\title{
A REMARK ON A MONOTONE SINGULAR FUNCTION
}

\section{H. KOBER}

1. In a former paper $^{1}$ I have dealt with a monotone singular function $\omega(t ; \alpha, \beta)$, where $\alpha>\beta>1, \beta$ an integer, $0 \leqq t<\infty$; and with a monotone jump-function $t=G(x ; \alpha, \beta)(0 \leqq x<\infty)$ such that $\omega(t)=x=G^{-1}$. These functions can be generalised. Let $\beta$ be any real number greater than one, $0 \leqq x<\infty$,

$$
\begin{aligned}
\gamma(x)=\gamma(x ; \alpha, \beta) & =\sum_{m=-\infty}^{\infty} \alpha^{-m}\left[\beta^{m} x\right]=\sum_{m \geqq-\log x / \log \beta} \alpha^{-m}\left[\beta^{m} x\right] ; \\
c_{\alpha, \beta} & =\lim _{0>\epsilon \rightarrow 0} 1 / \gamma(1-\epsilon),
\end{aligned}
$$

where $[u]$ denotes the greatest integer not exceeding $u(u \geqq 0)$, and set

$$
\begin{aligned}
\text { (i) } t & =g(x)=c_{\alpha, \beta} \gamma(x), \\
\text { (ii) } \Lambda(t) & =\Lambda(t ; \alpha, \beta)=x=g^{-1}(t) \quad(0 \leqq t<\infty) .
\end{aligned}
$$

It is not difficult to show that, in the special case when $\beta$ is an integer,

$$
\begin{aligned}
c_{\alpha, \beta}=\frac{(\alpha-\beta)(\alpha-1)}{\alpha(\beta-1)} ; g(x-0) \equiv G(x), \Lambda(t) & =\omega(t) \\
& (0 \leqq x, t<\infty) .
\end{aligned}
$$

The function $\omega(t ; \alpha, \beta)$ was a generalisation of the Cantor ternary set function $(\alpha=3, \beta=2){ }^{2}$ Thus the latter is representable in the simple form

$$
\begin{aligned}
\omega(t ; 3,2)=g^{-1}(t) ; \quad t=g(x)=2 \sum_{m=-\infty}^{\infty} 3^{-m-1}\left[2^{m} x\right] & \\
& (0 \leqq t, x<\infty) .
\end{aligned}
$$

Several of the results on $G(x)$ and $\omega(t)$ hold for the general case (compare (1.5), (2.1), (2.2) with previous results). Some of the proofs can be shortened owing to the concise form of $g(x)$ : and the result (2.5) on which (2.2) is based is even sharper than the corresponding one obtained previously.

Received by the editors September 11, 1951.

1 Trans. Amer. Math. Soc. vol. 67 (1949) pp. 433-450. The function $t=G(x)$ is $G(x)=(\alpha-\beta)(\beta-1)^{-1} \sum_{-\infty}^{\infty} a_{m, x} \alpha^{-m}$, where $a_{m, x}$ is the number of positive integers $n$ which are not divisible by $\beta$ and such that $n \beta^{-m}<x$; while $\omega(t)=x=G^{-1}(t)$.

${ }^{2} \mathrm{H}$. Lebesgue, Leçons sur l'integration, 2d ed., Paris, 1928. E. Hille and J. D. Tarmarkin, Amer. Math. Monthly vol. 36 (1929) pp. 255-264. 
Plainly $g(x)$ is discontinuous at the points $X_{\mu, \nu}=\mu \beta^{-\nu}(\mu=1,2, \cdots$; $\nu=0, \pm 1, \pm 2, \cdots)$ which are dense everywhere in $(0, \infty)$. Hence ${ }^{3}$ $g(x)$ is a strictly increasing jump-function, $\Lambda(t)$ is monotone, continuous, constant on the intervals of an open set of measure $a$ for $0 \leqq t \leqq a ;$ also

$$
\begin{aligned}
g(0) & =0=\Lambda(0), & g(1-) & =1=\Lambda(1) ; \\
g(x) & =\alpha g(x / \beta), & \Lambda(t) & =\beta \Lambda(t / \alpha) .
\end{aligned}
$$

2. We shall show that $\Lambda(t)$ is subadditive:4

$$
\Lambda(t+\tau) \leqq \Lambda(t)+\Lambda(\tau) \quad(t \geqq 0, \tau \geqq 0) ;
$$

and that, given $\beta_{0}>1$ and $M>0,5$

$$
g(x ; \alpha, \beta) \rightarrow x, \quad \Lambda(t ; \alpha, \beta) \rightarrow t
$$

as $\alpha-\beta \rightarrow 0$, uniformly for $\alpha>\beta \geqq \beta_{0}, 0 \leqq x \leqq M, 0 \leqq t \leqq g(M)$. Since $[u+v] \geqq[u]+[v](u \geqq 0, v \geqq 0), g(x+y) \geqq g(x)+g(y)(x \geqq 0, y \geqq 0)$ by (1.1). Consequently (2.1) is true. To prove (2.2) we take first $M<1$. For $0 \leqq x<1$ we have $\gamma(x)=\sum_{1}^{\infty} \alpha^{-m}\left[\beta^{m} x\right]$; hence

$$
\begin{aligned}
\frac{x \beta}{\alpha-\beta} & =\sum_{1}^{\infty} \alpha^{-m} \beta^{m} x \geqq \gamma(x) \geqq \sum_{1}^{\infty} \alpha^{-m}\left(\beta^{m} x-1\right) \\
& =\frac{x \beta}{\alpha-\beta}-\frac{1}{\alpha-1} .
\end{aligned}
$$

Multiplying by $c_{\alpha, \beta}$, taking $x \rightarrow 1$, and observing that $g(1-)=1$, we deduce that

$$
0 \leqq \frac{\beta c_{\alpha, \beta}}{\alpha-\beta}-1 \leqq \frac{c_{\alpha, \beta}}{\alpha-1} \leqq \frac{\alpha-\beta}{\alpha(\beta-1)} .
$$

From (2.3) we infer further that, for $0 \leqq x<1,0 \leqq \beta c_{\alpha, \beta} x /(\alpha-\beta)$ $-g(x) \leqq c_{\alpha, \beta} /(\alpha-1)$. Hence, taking $\beta c_{\alpha, \beta} /(\alpha-\beta)-1=u$, we have $-\left\{c_{\alpha, \beta} /(\alpha-1)-u x\right\} \leqq g(x)-x \leqq u x$,

$$
|g(x)-x| \leqq \max \left(u, \frac{c_{\alpha, \beta}}{\alpha-1}\right) \leqq \frac{\alpha-\beta}{\alpha(\beta-1)} \quad(0 \leqq x<1) .
$$

Consequently $(2.2)$ is true for $M<1$. Let now $M \geqq 1, n$ $=[\log M / \log \beta]+1, x^{\prime}=x \beta^{-n} ;$ then $M<\beta^{n} \leqq M \beta, 0 \leqq x^{\prime}<1, n$

${ }^{3}$ H. Kober, loc. cit., cf. 1.1.

4 Loc. cit., 1.4; here a different method of proof is needed.

${ }^{5}$ Loc. cit., 2.1. 
$\leqq \log M / \log \beta_{0}+1, g\left(x^{\prime}\right)<1$, and by $(1.5)$,

$$
\begin{aligned}
|g(x)-x| & =\left|g\left(\frac{x}{\beta^{n}}\right)-\frac{x}{\alpha^{n}}\right| \alpha^{n} \\
& \leqq\left|\left\{g\left(x^{\prime}\right)-x^{\prime}\right\} \beta^{n}+g\left(x^{\prime}\right)\left(\alpha^{n}-\beta^{n}\right)\right| \\
& \leqq M \beta\left|g\left(x^{\prime}\right)-x^{\prime}\right|+M n 2^{n-1}(\alpha-\beta) \quad\left(\beta_{0} \leqq \beta<\alpha \leqq 2 \beta\right) .
\end{aligned}
$$

Combining this with (2.5) we complete the proof.

3. By a very elementary theorem, any continuous function $f(x)$ $(0 \leqq x \leqq 1)$ is uniformly approximated by step-functions. By means of (2.2), we can find explicit step-functions of the kind required. ${ }^{6}$ Take, for instance,

$$
\begin{aligned}
& f_{n}(x)=f\left\{\frac{(\alpha-2)(\alpha-1)}{\alpha} \sum_{m=1}^{n^{2}} \alpha^{-m}\left[2^{m} x\right]\right\} \quad(0 \leqq x<1) \\
& f_{n}(1)=\lim _{x \rightarrow 1} f_{n}(x),
\end{aligned}
$$

where $\alpha=2+1 / n$. Then $f_{n}(x)$ is a step-function, and $f_{n}(x) \rightarrow f(x)$ as $n \rightarrow \infty$, uniformly in $(0,1)$. We omit the proof.

Another application of (2.2) is the approximation to functions by nearly analytic functions, i.e. by functions which in a domain are continuous and, at almost all points, analytic. It can be shown that any continuous function $f(x, y)\left(x^{2}+y^{2} \leqq 1\right)$ of two real variables is uniformly approximated by nearly analytic functions $H_{n}(z) \quad(|z|$ $=|x+i y| \leqq 1 ; n=1,2, \cdots)$. Given further $\phi(z)$, analytic and with bounded derivative for $|z|<1$, approximating $H_{n}(z)$ 's can be constructed such that $H_{n}^{\prime}(z)=\phi^{\prime}(z)$ p.p. and that, if $f(x, y)$ satisfies a Lipschitz condition, every $H_{n}(z)$ satisfies a Lipschitz condition also. Plainly these results are considerably more general than those on the approximation to analytic functions which we have proved previously. ${ }^{7}$

BIRMINGHAM, ENGLAND

- Cf. the result on approximation by functions with an infinity of jumps in loc. cit., end of 2.8 .

7 Loc. cit., 2.7. 